\title{
Asymptomatic throat carriage rate and antimicrobial resistance pattern of Streptococcus pyogenes in Nepalese school children
}

\author{
Dumre SP1, Sapkota K², Adhikari N², Acharya D ${ }^{2}$, Karki M³ , Bista S², Basnyat SR ${ }^{2}$, Joshi SK \\ ${ }^{1}$ National Public Health Laboratry, Teku, Kathmandu, Nepal, ${ }^{2}$ Department of Microbiology, Kantipur College of Medical \\ Science, Kathmandu, Nepal, ${ }^{3}$ Central Department of Microbiology, Tribhuwan University, Kirtipur, Nepal, ${ }^{4}$ Department \\ of Community Medicine, Kathmandu Medical College, Nepal
}

\begin{abstract}
Background: Streptococcus pyogenes or Group A streptococcus (GAS) causes several suppurative and non suppurative infections. In addition to pharyngitis and skin infections, GAS are also the causative agent of post-streptococcal infection syndromes such as acute rheumatic fever (ARF) and post-streptococcal glumerulonephritis (PSG). GAS frequently colonises in the throat of an asymptomatic person. Pharyngeal carriage rates of GAS among healthy school children vary with geographical location and seasons.

Objectives: We carried out this preliminary study to determine the throat carriage rate and antimicrobial resistance trend of Streptococcus pyogenes or Group A streptococcus (GAS) among the Nepalese school children.

Materials and methods: Four schools situated at different locations of Kathmandu valley were included in the study. Throat swabs from 350 students of age group 5-15 years were collected, immediately transported to the laboratory and were processed for $S$. pyogenes following standard microbiological procedures. Antimicrobial susceptibility testing of the isolates was performed by Kirby Bauer disc diffusion method following CLSI guidelines.

Results: S. pyogenes was isolated from 10.9\% (38/350) of the screened children. The GAS colonisation rate was statistically insignificant $(\mathrm{P}>0.05)$ with sex and age sub-groups, although the rate was slightly higher among girls and age sub-group 9-12 years. No significant difference in carrier rate was observed among different schools $(\mathrm{P}>0.05)$. All isolates were susceptible to azithromycin. No resistance was detected for penicillin and its derivative antibiotic ampicillin. Highest resistance rate was observed for cotrimoxazole $(71.0 \%)$ followed by chloramphenicol $(7.8 \%)$, ciprofloxacin (5.2\%) and erythromycin (5.2\%).

Conclusion: Antibiotic resistant GAS isolated from asymptomatic Nepalese school children is a public health concern. When screened and appropriately treated with antibiotics, carriers can be prevented from spreading of streptococcal infections in the school environment and the community. Preventing cross infections would ultimately reduce the incidence of life-threatening sequelae which are debilitating and difficult to treat. It is recommended to conduct regular screening and GAS surveillance in schools, and maintain rational use of antibiotics to minimise GAS carriage/infections and resistance.
\end{abstract}

Key words: Streptococcus pyogenes, Antibiotics Resistance, Throat carriage, Children, Nepal.

Ctreptococcus pyogenes or Group A streptococcus $\mathcal{N}$ (GAS) causes several suppurative and non suppurative infections and is responsible for a minimally estimated 616 million cases of throat infection (pharyngitis, tonsillitis) worldwide per year, and 111 million cases of skin infection (primarily non-bullous impetigo) in children of less developed countries ${ }^{1}$. Group A streptococci (GAS) are the most frequently isolated pathogens in acute pharyngo-tonsilitis cases in school-aged children causing approximately $20 \%$ of the pharyngitis cases ${ }^{2,3}$. In addition to pharyngitis and skin infections, GAS are also the causative agent of post-streptococcal infection syndromes such as acute rheumatic fever (ARF) and post-streptococcal glumerulonephritis (PSG). The majority of acquired heart diseases in children, particularly in the developing countries are caused by ARF and PSG. GAS frequently colonises in the throat of an asymptomatic person ${ }^{2}$. Pharyngeal carriage rates of GAS among healthy school children vary with geographical location and seasons ${ }^{1}$. Among asymptomatic children, carriage rates of 15$20 \%$ have been reported in several studies ${ }^{4}$.

\footnotetext{
Correspondence

Shyam Prakash Dumre

Microbiologist

National Public Health Laboratory, Teku, Kathmandu, Nepal

E-mail: shyamprad@hotmail.com
} 
Since early 1980s there has been re-emergence in the incidence of invasive $S$. pyogenes infections and rheumatic heart disease all over the world and hence throat carriage has assumed of great importance ${ }^{5}$. Rheumatic heart disease is still prevalent in developing countries, particularly among the children who live in communities that do not have adequate treatment programmes. Beta-lactam antibiotics (mainly Penicillins) and macrolides have been conventionally used to treat $S$. pyogenes infections ${ }^{6}$. The resistance of $S$. pyogenes to macrolides has been reported and is increasing in most of the countries ${ }^{7,8}$ and there were no report of penicillin resistance although isolates with rising minimum inhibitory concentration (MIC) to penicillin are increasing 9 . Studies on antibiotic resistance among isolates from pharyngeal carriers could provide important data on resistance profiles of strains circulating in the community.

In Nepal, there is lack of base line literature on the carriage rates and resistance patterns of GAS in school children. The present study was undertaken to understand throat carriage rate and antimicrobial susceptibility pattern of GAS among children of different schools within Kathmandu valley. In view of the increasing number of drug resistance pattern among GAS, we also attempted to assess the antibiotic resistance pattern among isolated GAS from the studied samples.

\section{Materials and methods}

Study site: The study population included Nepalese school children from four schools situated at different locations of Kathmandu valley. Laboratory Analysis was performed at Department of Microbiology, Kantipur College of Medical Sciences, Kathmandu, Nepal.

Study population: During February through April 2007, prospective collection of the clinical and microbiological data was completed from healthy school children who were asymptomatic for throat infection. A total of 350 asymptomatic children were included of which $45.7 \%$ were boys. The median age of the children was 9.6 years (range, 5-15 years). Children with throat infection or any related sign and symptoms of pharyngitis were excluded from the study. The asymptomatic/healthy and symptomatic children were clinically differentiated by a medical practitioner after clinical examination. Students having past medical history, including antimicrobial therapy and/or hospital attendance, were obtained by formally requesting and collecting medical records from their parents/guardians. Those who received antimicrobial therapy or who had suffered from GAS in the previous three months were excluded from the study. With the recent report of GAS colonisation in lower age group, we divided the age into three sub-groups namely 5 to 8,9 to 12 and 13 to 15 years. Within this narrow age range, we wished to see if making three sub-groups has any variation in GAS carriage.

Specimen collection: Study permission was secured from Department of Microbiology, Kantipur College of Medical Science and the Management Board of participating schools. After institution permission, we forwarded a letter explaining objectives of our study to children's parents/guardians. After getting written informed consent from guardians/parents or their care takers with legal custody of the children, pharyngotonsillar specimens were obtained with sterile cotton tipped swab. All the swabs were aseptically transferred to Stuart's transport medium and transported to the laboratory within two hours of collection.

Bacteriological methods: The swabs obtained in transport medium were cultured in enriched solid agar media plates $(5 \%$ sheep blood agar, and chocolate agar) and enrichment broth (Brain Heart Infusion, BHI) immediately upon receipt at the microbiology laboratory of Kantipur College of Medical Science. Inoculated culture media were incubated at $37^{\circ} \mathrm{C}$ in $5 \%$ carbon dioxide and examined at 24 and 48 hours. Plates without beta haemolytic colonies after 24 hours incubation were re-subcultured on sheep blood agar and chocolate agar plates from the overnight incubated BHI broth. All the plates with beta-haemolytic colonies were microbiologically processed and GAS were identified by conventional methods (colony morphology, haemolysis pattern, catalase test, Gram stain and morphological observation). Streptococcus pyogenes was further identified by observing its sensitivity towards 0.04 units of bacitracin disc tested on sheep blood agar plate.

Antimicrobial susceptibility testing: Antimicrobial susceptibility testing of the isolates was performed by Kirby Bauer disc diffusion method following clinical and laboratory standards institute (CLSI) recommended interpretive criteria $^{10}$. Streptococcus pneumoniae ATCC 49612 was used as the quality control strain. The following antibiotics were tested for all the conformed isolates: Penicillin (10 U), Ampicillin $(10 \mu \mathrm{g})$, Ciprofloxacin $(5 \mu \mathrm{g})$, Azithromycin $(15 \mu \mathrm{g})$; Erythromycin $(15 \mu \mathrm{g})$, Cotrimoxazole $(1.25 / 23.75 \mu \mathrm{g})$ and Chloramphenicol $(30 \mu \mathrm{g})$.

Statistical analysis: Data were coded and sorted in a standard format in MS-Excel sheet. Randomised complete block design (RCBD) and 2 X 3 factorial treatment structures was used to determine the effect of age groups and sex of school children on carriage rate of GAS. There were three age sub-groups and four schools. Age groups and sex were considered as factors and schools were treated as block in Statistical 
Analysis System (SAS) design. Statistical analysis was performed using latest SAS software, version 9.2. The relevant SAS outputs are depicted in table 1, 2 and 3.

Results

S. pyogenes was isolated from $10.9 \%$ (38/350) of the screened children. Isolation rate was slightly higher among girls $(12.5 \%)$ than boys $(9.4 \%)$, however it was statistically insignificant $(\mathrm{P}>0.05)$. Although the carriage rate was higher in the age sub-group 9-12 (tables 1, 3), the difference was statistically insignificant $(\mathrm{P}>0.05)$.
Similarly, no significant difference in carriage rate was found among the four different schools studied (tables 2, 3).

Of the 38 S. pyogenes strains isolated, $27(71.0 \%)$ were found to be resistant to Cotrimoxazole. Resistance to Chloramphenicol, ciprofloxacin and Erythromycin was in $3(7.8 \%), 2(5.2 \%), 2(5.2 \%)$ isolates respectively. No resistance was detected among Penicillin and its derivative (Penicillin $G$ and Ampicillin). Similarly, Azithromycin was also found to be $100 \%$ susceptible.

Table 1: Age wise distribution of throat carriage of $S$. pyogenes

\begin{tabular}{|l|l|c|c|c|c|c|c|}
\hline \multirow{2}{*}{$\begin{array}{l}\text { Age sub- } \\
\text { group }\end{array}$} & \multirow{2}{*}{$\begin{array}{l}\text { Number of students } \\
\text { screened }\end{array}$} & \multicolumn{2}{|c|}{ GAS positive } & \multirow{2}{*}{$\begin{array}{c}\text { Mean \% } \\
\text { of GAS }\end{array}$} & \multicolumn{2}{|c|}{$\begin{array}{c}\mathbf{9 5} \text { Confidence } \\
\text { Interval }\end{array}$} & \multirow{2}{*}{ P value } \\
\cline { 3 - 4 } & & $\mathbf{n}$ & $\mathbf{\%}$ & & Lower & Upper & \\
\hline $5-8$ & 110 & 12 & 10.9 & 7.9 & 4.5 & 11.2 & \\
\hline $9-12$ & 125 & 15 & 12.0 & 9.9 & 6.5 & 13.2 & $>0.05$ \\
\hline $13-15$ & 115 & 11 & 9.6 & 7.2 & 3.9 & 10.6 & \\
\hline Total & $\mathbf{3 5 0}$ & $\mathbf{3 8}$ & $\mathbf{1 0 . 9}$ & & & \\
\hline
\end{tabular}

Table 2: School wise distribution of throat carriage of S. pyogenes

\begin{tabular}{|c|c|c|c|c|}
\hline \multirow{2}{*}{ School } & \multirow{2}{*}{ Number of children screened } & \multicolumn{2}{|c|}{ GAS positive } & \multirow{2}{*}{ P value } \\
\hline & & $\mathbf{n}$ & $\%$ & \\
\hline Secondary School located at Sitapaila & 95 & 9 & 9.5 & \multirow{4}{*}{$>0.05$} \\
\hline Secondary School located at Maitidevi & 85 & 8 & 9.4 & \\
\hline Secondary school located at Swyambhu & 80 & 9 & 11.2 & \\
\hline Primary school located at Kirtipur & 90 & 12 & 13.3 & \\
\hline Total & 350 & 38 & 10.9 & \\
\hline
\end{tabular}

Table 3: Analysis of variance (ANOVA) on age subgroups and sex of four schools children

\begin{tabular}{|l|c|c|c|l|}
\hline Source of variance & DF & F-value & P-value & Remarks \\
\hline Age & 2 & 0.64 & 0.64 & No significant age effect \\
\hline Sex & 1 & 0.99 & 0.99 & No significant sex effect \\
\hline Age by sex & 2 & 1.59 & 1.59 & No significant age by sex interaction \\
\hline School & 3 & 0.53 & 0.53 & No significant school effect \\
\hline
\end{tabular}

\section{Discussion}

We report the notable proportion of pharyngeal carriage by group A streptococci among healthy children from different schools of Nepal. GAS throat carriage is an important public health issue, as the infection often leads to post streptococcal sequelae and individuals colonised with GAS can serve as a source of spread of infections to other individuals in the community. An overall $10.9 \%$ of asymptomatic school children, irrespective of the sex and age sub-groups ( $\mathrm{P}>0.05)$, were found to be colonised with GAS. Due to unavailability of published literature on GAS carriage from Nepal, it is difficult to estimate the increasing or decreasing trend of the condition.
The present throat carriage rate of GAS found in Nepalese school children is in accordance with the findings of other studies carried out in many parts of the world. Few studies in different geographical location, settings and countries, however, have shown a different carriage rate. This is because the relative incidence of disease caused by $S$. pyogenes varies throughout the world, in accordance with season and age group ${ }^{1,11}$. In general, the prevalence of carriage of GAS in healthy individuals decreases with age. In a report, the prevalence of streptococci group A was 6\% in all the age groups studied $^{12}$, with $8.6 \%$ in carriers among healthy children ${ }^{13}$ and $12.2 \%$ on school children ${ }^{14}$. Another study reports 
low prevalence of GAS in healthy individuals before the age of 3 years and in adults above 16 years. But on the contrary, the same study reports highest prevalence rate of GAS in the age group 3-15 years ${ }^{11}$. In similar studies conducted in turkey, the rate of GAS carriage in asymptomatic school children varied from 2 to $46 \%{ }^{1,15}$. In the present study, though statistically not significant, the age sub-group 9-12 years was the most susceptible group for throat carriage of GAS, followed by age subgroups 5-8 and 13-15 years respectively. Age sub-group wise difference was not significant in our study since we had a narrow age range and focused only in children population from 5-15 years of age. Few studies have reported the age group variation ${ }^{1,11}$ which could be due to analysis of all age groups including adults.

We found no significant variation of carriage rate with different schools as well. A study carried out in Turkish school children showed the variation in the carriage rate of GAS in two different schools children; the overall carriage rate was $17 \%$, with $6 \%$ in students from school in impoverished area and $28 \%$ in students from school in suburban area ${ }^{16}$. No significant variation with schools in our study could be due to the reason that all schools were almost similar in every aspect. Moreover, the schools included in this study were not geographically diverse and hence a similar rate was observed.

There are several mechanisms suggested for colonisation of GAS in pharynx ${ }^{17}$ and the colonisation may turn into clinical infections under certain circumstances ${ }^{18}$. A study on environmental health effects of brick kilns in Kathmandu valley showed high prevalence of tonsillitis $(4.17 \%)$ and acute pharyngitis $(4.08 \%)$ in school children residing nearby brick kilns ${ }^{19}$. We assume that air pollution may enhance the infection in GAS colonised children. However, confirmatory relation can only be established when both epidemiological and etiological investigations are conducted to show if air pollution enhances GAS related throat infection in children.

Due to the more rapid acquisition of resistance, obtaining appropriate treatment for severe invasive streptococcal infections is now a major challenge in many regions of the world. Our study showed a quite high resistance rate $(71.0 \%)$ towards Cotrimoxazole which is one of the commonly used drugs to treat children infected with various diseases in Nepal. Such a high level of resistance in a commonly used drug possesses a potential risk for spread of resistance to other microorganisms as well. None of the strains we isolated was found resistant with penicillin and Ampicillin. Penicillin and its derivative remained the drug of choice for streptococcal pharyngitis with stable minimum inhibitory concentration (MIC) during the last 70 years $^{9}$. However, reports of a rising
MIC or diminished susceptibility to penicillin have been published in recent years ${ }^{9,20}$. In Nepal, penicillin derivatives are among the easily available antibiotics even in the sub-health post level where culture facility is not available. Although none of the literature reviewed has reported Penicillin resistant in GAS till date, empirical therapy based on penicillin and its derivatives for most of the respiratory infections in the peripheral settings of developing countries like Nepal, boots up the possible emergence of resistance towards this valuable drug in the future. Resistance to both ciprofloxacin and erythromycin was found in $5.2 \%$ isolates. Macrolide resistant $S$. pyogenes isolates are increasing in some parts of the world and $20-30 \%$ resistance is being reported in some countries ${ }^{21}$. We found Azithromycin as 100\% susceptible drug against this pathogen in addition to Penicillin and Ampicillin. Now-a-days, because of its effectiveness and short course therapy, Azithromycin is commonly prescribed drug in Nepal for respiratory tract infections. However, its high frequency of prescription and possible irrational use may help to evolve Azithromycin resistant strain in the future. Therefore, before changing the therapeutic options, it is highly recommended to review the existing antimicrobials and their regimen based on their potency to save the valuable drugs for critical clinical conditions.

\section{Conclusion}

The present preliminary study provides the base-line information on the GAS carriage rate and resistance trend among healthy school children. It is highly emphasised that GAS carriage surveillance needs to be initiated and established in a large scale population to estimate the national scenario. As the limitation, our study was restricted to schools of Kathmandu valley. Therefore, the carriage rate could be different for the schools of rural areas (outside Kathmandu valley). To draw the clear-cut picture, studies among both healthy and symptomatic children of different geographical locations needs to be conducted. More detailed study is also required to establish the relationship between carriage, acute sore throat, subsequent anti-streptolysin-O titre levels, and their relationship to the post streptococcal sequelae. When screened and appropriately treated with antibiotics, pharyngeal carriers can be prevented from spreading of streptococcal infections in the community. This would ultimately reduce the incidence of lifethreatening post-infectious sequelae such as ARF and PSG, which are debilitating and difficult to treat. Spread of resistance could be minimised by appropriate treatment guidelines, rational use of antimicrobials, review of existing antimicrobial therapy with regimen and creating awareness on antimicrobial resistance. 
Acknowledgement

We wish to thank the participating schools' teachers and guardians for their consent and cooperation during the entire period of our study. We would also like to thank undergraduate and graduate students of Kantipur College of Medical Science for helping in sample collection and Mr. Raju Lama for assisting in culture and sensitivity tests. Special thanks go to Mr. Achyut Adhikari for providing constructive feedback on research design and statistical analysis using SAS.

\section{References}

1. Carapetis JR, Steer, AC, Mulholland EK, Weber M. The global burden of group A streptococcal diseases. Lancet Infect Dis. 2005;5:685-94.

2. Martin JM, Green M, Barbadora KA, Wald ER. Group A streptococci among school-aged children: clinical characteristics and the carrier state. Pediatrics. 2004;114(5):1212-9.

3. Dubosa KC. Group A streptococcal pharyngitis. Prim Care Updates Ob Gyns. 2002;9:222-5.

4. Bisno AL, Stevens DL. Streptococcus pyogenes. In: Mandell GL, Bennett JE and Dolin R (editors). Principle and practice of infectious disease. 5th edition. New York: Churchill Livingstone; 2000.p.2107-17.

5. Kaplan EL, Johnson DR, Cleary PP. Group A streptococci serotypes isolated from patients and sibling and contacts during the resurgence of rheumatic fever in United States in the mid 1980s. J Infect Dis. 1989;159:101-3.

6. Horn DL, Zabriskie JB. Why have group A streptococci remained susceptible to penicillin? Report on a symposium. Clin Infect Dis. 1998;26:1341-5.

7. Cornaglia G, Ligozzi M, Mazzariol A, Masala L, Lo Cascio G, Orefici G, et al. Resistance of Streptococcus pyogenes to erythromycin and related antibiotics in Italy. Clinical Infectious Diseases. 1998;27(Suppl 1):87-90.

8. Perez-Trallero E, Urbieta M, Montes $M$, Ayestaran I, Marimon JM. Emergence of Streptococcus pyogenes strains resistant to erythromycin in Gipuzkoa, Spain. European Journal of Clinical Microbiology and Infectious Diseases. 1998;17:25-31.

9. Capoor MR, Nair D, Monorama D, Batra K, Agrawal P. Resistance to Erythromycin and rising penicillin MIC in Streptococcus pyogenes. Ipn J Infec Dis. 2006;59:334-6.

10. CLSI, NCCLS. Clinical and Laboratory Standard Institute: Performance standards for antimicrobial disk susceptibility test: approved standard. M2-A9. 9th edition. Wayne: CLSI, NCCLS; 2006.

11. Gunnarsson RK, Holm SE, Söderström M. The prevalence of beta-haemolytic streptococci in throat specimens from healthy children and adults. Implications for the clinical value of throat cultures. Scand J Prim Health Care. 1997;15(3):149-55.

12. González-Lama Z, González JJ, Lupiola $\mathrm{P}$, Tejedor MT. Carriers of beta hemolytic streptococci from groups A, B, and C among schoolchildren in Las Palmas. Enferm Infecc Microbiol Clin. 2000;18(6):271-3.

13. Braito A, Galgani I, Mohammed MR, Iozzi C, Ame SM, Haji HS, et al. Epidemiology of streptococcus group A in school aged children in Pemba. East Afr Med J. 2004;81(6):307-12.

14. Takeuchi T, Kawakita S. A follow-up study of throat carriers of streptococci among schoolchildren in Otsu City. Jpn Circ J. 1985;49(12):1254-7.

15. Metintas S, Kalyoncu C, Etiz S, Kiraz N, Unsal N. Prevalence of group A beta haemolytic Streptococcus carriers in primary school students of Cifteler, Turkey. Anatolia Med J. 1991;13:17-27.

16. Altindis M, Derekoy FS, Ceri A. Turkish primary school students as carriers of group A beta haemolytic streptococci and susceptibility of strains to penicillin and erythromycin. $\mathrm{J}$ Chemother. 2001;13:444-5.

17. Cywes C, Stamenkovic I, Wessels MR. CD44 as a receptor for colonization of the pharynx by group A Streptococcus. J. Clin. Invest. 2000;106 (8): 995-1002.

18. Terao Y. Possible mechanisms related to development of severe Streptococcus pyogenes infection . J. Oral Biosci. 2008; 50: 89-7.

19. Joshi SK, Dudani I. Environmental health effects of brick kilns in Kathmandu valley. Kathmandu Univ Med J. 2008;6(1):3-11.

20. Amabile-Cuevas CF, Harmida-Escobedo C, Vivar R. Comparative in vitro activity of moxifloxacin by E-test against streptococcus pyogenes. Clin Infec Dis. 2001;32:S30-S32.

21. Canton R, Loza E, Morosini MI, Baquero F. Antimicrobial resistance amongst isolates of Streptococcus pyogenes and Staphylococcus aureus in the PROTEKT antimicrobial surveillance programme during 1999-2000. J Antimicrob Chemother. 2002;50(Suppl 1):924. 